\title{
Montagem e aplicação de modelo de baixo custo de dissecção venosa
}

\author{
Making up and application of a low cost model of venous dissection
}

\section{Carlos Magno Queiroz da Cunha ${ }^{1}$, José Alberto Guilherme Frota Júnior ${ }^{1}$, James Dantas Ferreira ${ }^{1}$, Giovanni Troiani Neto ${ }^{1}$, Diego Freitas Félix ${ }^{1}$, Francisco Julimar Correia de Menezes ${ }^{2}$}

Cunha CMQ, Frota Júnior JAG, Ferreiras JD, Troiani Neto G, Félix DF, Menezes FJC. Montagem e aplicação de modelo de baixo custo de dissecção venosa / Making up and application of a low cost model of venous dissection. Rev Med (São Paulo). 2017 out.-dez.;96(4):220-4.

\begin{abstract}
RESUMO: Objetivo: Desenvolver e aplicar modelo de baixo custo para treinamento de dissecção venosa para acadêmicos de Medicina. Métodos: O modelo foi elaborado com custo fixo de 35 reais (10,5 dólares) e mais 50 centavos a cada reposição, tendo sido aprovados por 6 cirurgiões vinculados ao curso de Medicina da Universidade de Fortaleza e utilizados durante curso teórico prático. Os alunos responderam a um teste pré e pós-atividade, foram avaliados durante a prática através de check list e responderam questionário de percepção sobre o modelo. Resultados: Foi notado crescimento teórico, porém, percebeu-se a necessidade de mais treinamento procedural, o que é viável com nosso modelo de baixo custo. Em relação aos questionários de percepção, 91,95\% dos alunos concordaram que os modelos mantinham boa correlação anatômica, $89,96 \%$ que o material utilizado é de boa qualidade, $95,40 \%$ que o modelo permitiu o aprendizado da dissecção venosa e $96,55 \%$ que o modelo pode ser utilizado para ensino do procedimento, números que reafirmam a eficácia e viabilidade do modelo. Conclusões: É possível a criação de modelo realista, de boa qualidade e viável para o ensino, apenas com materiais de baixo custo para treinamento de dissecção venosa.
\end{abstract}

Descritores: Materiais de ensino; Dissecação/educação; Treinamento por simulação; Estudantes de medicina.

\begin{abstract}
Objective: We aim to develop and apply a low cost model for venous dissection training for medical students. Methods: The model was elaborated with a fixed cost of 35 reais (10,5 dollars) and 50 cents at each replacement, and was approved by 6 surgeons tied to the Medicine course of the Universidade de Fortaleza and used during practical theoretical course. The students answered a pre and post activity test, were evaluated during the practice through check list and answered the perception questionnaire about the model. Results: We noticed a theoretical growth, however, we noticed the need for more procedural training, which is feasible with our low cost model. Regarding the perception questionnaires, $91.95 \%$ of the students agreed that the models maintained good anatomical correlation, $89.96 \%$ that the material used was of good quality, $95.40 \%$ that the model allowed the learning of the venous dissection and $96.55 \%$ that the model can be used for teaching the procedure, numbers that reaffirm the effectiveness and feasibility of the model. Conclusions: It is possible to create a realistic model, of good quality and feasible for teaching, only with low cost materials for venous dissection training.
\end{abstract}

Keywords: Teaching materials; Dissecation/education; Simulation training; Students, medical.

Apresentado no XXXII Congresso Brasileiro de Cirurgia, São Paulo, SP, 28/04 a 01/05/2017.

1. Universidade de Fortaleza, Ceará, CE, BR. Acadêmicos de Medicina. E-mail: carlosmagnoqc@gmail.com, alberto.jr.frota@hotmail. com,jamess-93@hotmail.com, giovannitroianin@gmail.com, diegofelix.med@gmail.com

2. Universidade de Fortaleza, Ceará, CE, BR. Cirurgião Digestivo e Professor Associado da UNIFOR. E-mail: julimarmd@gmail.com. Endereço para correspondência: R. Des. Floriano Benevides Magalhães, 221 - Edson Queiroz, Fortaleza, CE, BR. CEP: 60811-905. 


\section{INTRODUÇÃO}

A s práticas cirúrgicas têm como um de seus pilares a aquisição de habilidades e técnicas. Diante disso, o uso de modelos sintéticos de baixo custo procura sanar os problemas impostos pelo tempo limitado para conciliar teoria com a prática e ultrapassar as dificuldades na obtenção de recursos humanos e materiais, além de distanciar-se das conhecidas polêmicas que envolvem o uso de animais em ensino e pesquisa ${ }^{1-5}$.

A dissecção venosa consiste na dissecção, isolamento, cateterismo e na maioria das vezes ligadura distal de uma veia, sendo realizado sob anestesia local. Para isso, podem ser utilizadas inúmeras veias superficiais como a veia basílica e as veias safenas ou profundas como a braquial. Esse procedimento está indicado principalmente em situações emergenciais onde não se consegue obter acesso venoso periférico prontamente, sendo assim um dos objetivos do curso do ATLS ${ }^{\circledR 6-9}$.

Diante do atual contexto evolutivo na formação médica citado anteriormente e da importância do treinamento prático na execução de tal técnica, a Liga de Cirurgia Geral da Universidade de Fortaleza (LICIG - UNIFOR) desenvolveu modelo sintético de baixo custo para treinamento de dissecção venosa em veia basílica.

\section{MATERIAL E MÉTODOS}

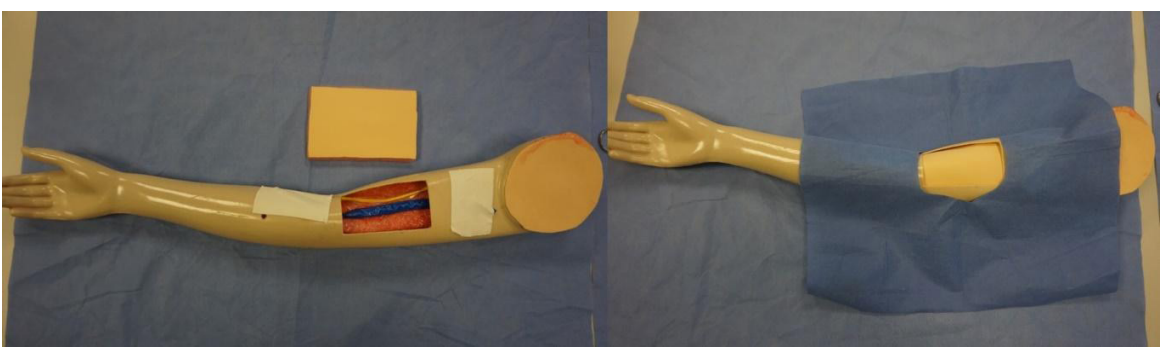

Figura 1. Modelo sendo montado (esquerda) e modelo pronto para realização do procedimento (direita)

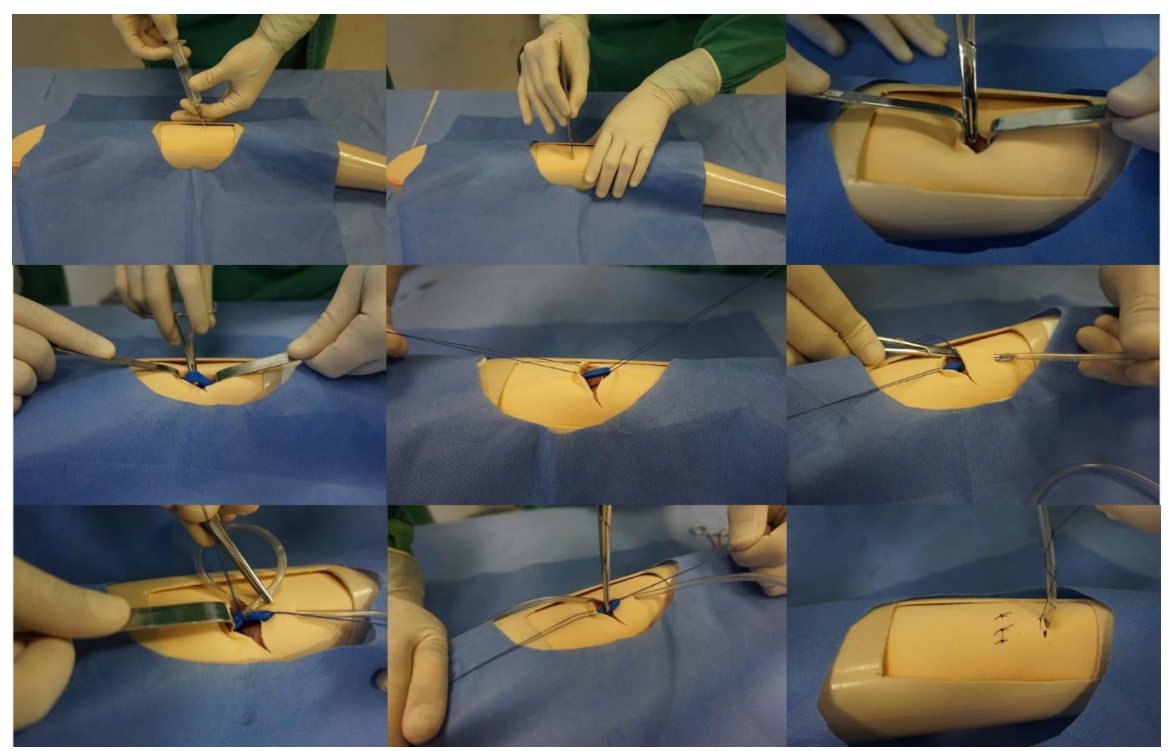

Figura 2. Realização do procedimento de dissecção venosa no modelo de 35 reais (aproximadamente 10,5 dólares) e a cada reposição, são gastos 0,50 centavos. Sua montagem foi feita em um manequim comercial de braço onde foi realizado um corte de formato retangular de dimensões 9,5 x $5,5 \mathrm{~cm}$ próximo à fossa cubital, que funcionará como via de acesso. Outros dois orifícios de formato circunferencial e de raio aproximado de $1 \mathrm{~cm}$ foram realizados, o primeiro mais proximal ao corte retangular e o segundo mais distal a este. Estes orifícios funcionam como local de suporte para fixação da "veia basílica" de nosso modelo.

Os músculos bíceps e tríceps braquial foram confeccionados a partir da cobertura de espuma flocada com papel filme de modo que a estrutura final tivesse um formato fusiforme. Foi usado corante acastanhado na espuma para melhor identificar a estrutura. Já a veia basílica foi reproduzida com uso de balão palito azul, preenchidos com corante vermelho para simular o sangue venoso. Também foi usado um fio elétrico de coloração amarelada para simular o nervo cutâneo medial.

Para preencher o corte retangular e simular a pele e o subcutâneo, foi usado folha E.V.A. de coloração semelhante e uma camada de espuma de estofado. Ao final de cada procedimento é substituída a pele, subcutâneo e veia em aproximadamente 1 minuto. 


\section{Aplicação}

Após criação do modelo pelos acadêmicos e orientador da LICIG, o modelo foi apresentado e testado por seis cirurgiões vinculados ao curso de Medicina da UNIFOR que avaliaram e aprovaram o modelo para uso.

Esses manequins foram utilizados durante curso teórico prático de Cirurgia realizado na UNIFOR, onde durante quatro horas os alunos de graduação assistiram aula teórica, demonstração no manequim e realizaram o procedimento. Antes da aula, os alunos responderam a um questionário teórico (pré-atividade) sobre dissecção venosa de cinco questões fechadas e voltaram a respondê-lo após toda a atividade (pós-atividade). Durante a realização do procedimento, os alunos eram avaliados através de check list padronizado (Quadro 1) e ao final responderam questionário likert de percepção sobre o modelo testado.

Todos os dados dos questionários foram analisados através do programa IBM SPSS v.22. A pesquisa foi aprovada pelo Comitê de Ética em Pesquisa da Universidade de Fortaleza (UNIFOR), CAAE 62192216.7.0000.5052, parecer 1.858.030.

\section{RESULTADOS}

O modelo só foi implementado e utilizado no curso supracitado após avaliação e aprovação por seis professores com formação na especialidade de Cirurgia do curso de
Medicina da UNIFOR, conforme descrito na Tabela 1.

Ao final dos dois cursos, 87 alunos seguiram a metodologia proposta. As características e os rendimentos deles se encontram nas Tabelas 2 e 3, respectivamente.

Quadro 1. Check list utilizado para avaliação dos alunos

\begin{tabular}{|l|l|l|}
\hline Etapa & Sim (1) & Não (0) \\
\hline $\begin{array}{l}\text { 1. Antissepsia e aposição de } \\
\text { campo. }\end{array}$ & & \\
\hline 2. Anestesia local. & & \\
\hline 3. Incisão cutânea. & & \\
\hline 4. Dissecção por planos. & & \\
\hline 5. Identificação da veia basílica. & & \\
\hline $\begin{array}{l}\text { 6. Reparar a veia, proximal e } \\
\text { distalmente com fio de seda. }\end{array}$ & & \\
\hline 7. Teste de tração. & & \\
\hline 8. Ligadura distal da veia. & & \\
\hline 9. Incisão da veia. & & \\
\hline 10. Inserção do cateter. & & \\
\hline $\begin{array}{l}\text { 11. Ligadura do coto proximal } \\
\text { sobre o cateter. }\end{array}$ & & \\
\hline 12. Síntese da pele. & & \\
\hline 13. Fixação do cateter. & & \\
\hline 14. Curativo. & & \\
\hline
\end{tabular}

Tabela 1. Questionário de percepção em escala Likert aplicada em cirurgiões vinculados à graduação de Medicina da UNIFOR

\begin{tabular}{|c|c|c|c|c|c|}
\hline Questionamento & CT & $\mathbf{C P}$ & $\mathbf{I N}$ & DP & DT \\
\hline O modelo mantém boa correlação anatômica? & $66,6 \%$ & $33,4 \%$ & $0 \%$ & $0 \%$ & $0 \%$ \\
\hline O material utilizado é de boa qualidade? & $66,6 \%$ & $0 \%$ & $16,67 \%$ & $16,67 \%$ & $0 \%$ \\
\hline O modelo permite o aprendizado da dissecção venosa? & $83,33 \%$ & $0 \%$ & $16,67 \%$ & $0 \%$ & $0 \%$ \\
\hline O modelo pode ser usado para o ensino do procedimento? & $100 \%$ & $0 \%$ & $0 \%$ & $0 \%$ & $0 \%$ \\
\hline
\end{tabular}

CT: Concordo Totalmente; CP: Concordo parcialmente; IN: Indiferente; DP: Discordo parcialmente; DT: Discordo totalmente.

Tabela 2. Características dos estudantes $(n=87)$ que participaram do estudo

\begin{tabular}{l|l|l|l}
\hline Característica & Total & Característica & Total \\
\hline Sexo n (\%) & & Semestre n (\%) & \\
\hline Masculino & $45(51,7 \%)$ & $1-4$ & $62(71,3 \%)$ \\
\hline Feminino & $42(48,3 \%)$ & $5-8$ & $25(28,7 \%)$ \\
\hline Idade & & Experiência prévia n (\%) & \\
\hline Média \pm desvio padrão & $19,98 \pm 1,73$ & Nenhuma & $39(44,8 \%)$ \\
\hline IES* n (\%) & & Teórico-prático & $11(12,6 \%)$ \\
\hline UNIFOR & $61(70,1 \%)$ & & \\
\hline Outras 6 IES & $16(29,9 \%)$ & & \\
\hline
\end{tabular}

*Instituição de Ensino Superior 
Cunha CMQ, et al. Montagem e aplicação de modelo de baixo custo de dissecção venosa.

Tabela 3 - Rendimento dos alunos nas avaliações propostas

\begin{tabular}{l|c}
\hline Avaliação & Rendimento \\
\hline Questionário teórico & \\
\hline $\begin{array}{l}\text { Média pré-atividade } \pm \text { desvio } \\
\text { padrão }\end{array}$ & $3,83 \pm 2$ \\
\hline $\begin{array}{l}\text { Média pós-atividade } \pm \text { desvio } \\
\text { padrão }\end{array}$ & $7,21 \pm 1,51$ \\
\hline Crescimento médio & $88,25 \%$ \\
\hline Check List & $84 \pm 13,95$ \\
\hline Média \pm desvio padrão & $42(48,3 \%)$ \\
\hline Contaminou n (\%) & 1084 segundos \pm 247 \\
\hline Tempo médio \pm desvio padrão
\end{tabular}

Já em relação ao questionário de percepção, idêntico ao respondido pelos professores cirurgiões do curso de Medicina da UNIFOR (Tabela 1), 91,95\% dos alunos concordaram em algum nível que os modelos mantinham boa correlação anatômica e $89,96 \%$ que o material utilizado é de boa qualidade. No quesito ensino e aprendizagem, $95,40 \%$ concordaram que o modelo permitiu o aprendizado da dissecção venosa e $96,55 \%$ que o modelo pode ser usado para o ensino do procedimento.

\section{DISCUSSÃO}

Percebe-se por meio dos resultados das avaliações pré e pós-atividade que os alunos tiveram um considerável crescimento teórico no assunto, demonstrando a eficácia nesse âmbito. Porém, em relação ao meio procedimental, percebe-se que há a necessidade de mais treinamento, o que é viável com nosso modelo, já que os materiais que utilizamos são de fácil acesso e de baixo custo, podendo ser reproduzidos facilmente.

Também é importante ressaltar que apesar dos alunos terem realizado a dissecção venosa em aproximadamente 18 minutos e $48,3 \%$ dos alunos contaminarem o procedimento, a média do check list foi acima de $80 \%$ o que demonstra um dos objetivos centrais do treinamento, que é a memorização do passo a passo do procedimento, sendo uma importante ferramenta durante a curva de aprendizado no procedimento. Dessa maneira, a prática em modelo sintético de baixo custo produz ambiente realístico, mas protegido, evitando potenciais iatrogenias presentes durante o aprendizado in vivo e embargos relacionados a questões éticas, financeiras e legais que acompanham outros métodos de ensino prático ${ }^{10-12}$.

Também encontramos limitações no modelo, como a reprodução fidedigna do teste de tração (usado para diferenciar veia de artéria) que apesar de ser realizado, o modelo não se comporta como uma veia (colaba quando tracionada a parte distal) já que não existe fluxo contínuo. Nesse manequim o cateter deve ser curto devido o comprimento do balão. É importante ressaltar que mesmo diante dessas intempéries, o modelo demonstra por completo toda a técnica de dissecção venosa da veia basílica, podendo ser expandidas para outros sítios de dissecção, apenas mudando as referências anatômicas ${ }^{13-15}$.

\section{CONCLUSÃO}

Desse modo, percebe-se que é possível a criação de modelo realista, de boa qualidade e viável para o ensino, apenas com materiais de baixo custo.

Participação dos autores no texto: Cunha CMQ. Idealizador do modelo e do trabalho, participou da montagem e aplicação do modelo e análise estatística, redação e revisão do manuscrito. Frota Júnior JAG. Participou da montagem e aplicação do modelo e redação do manuscrito; Ferreiras JD. Participou da montagem e aplicação do modelo e redação do manuscrito; Troiani Neto G. Participou da montagem e aplicação do modelo e redação do manuscrito; Félix DF. Participou da montagem e aplicação do modelo e redação do manuscrito; Menezes FJC. Orientador do trabalho, participou da montagem e aplicação do modelo e análise estatística, redação e revisão do manuscrito.

\section{REFERENCIAS}

1. Amoretti R. A educação médica diante das necessidades sociais em saúde. Rev Bras Educ Med. 2005;29(2):136-46.

2. Gomes AP, Rego S. Transformação da educação médica: é possível formar um novo médico a partir de mudanças no método de ensino-aprendizagem? Rev Bras Educ Med. 2011;35(4):557-66. http://dx.doi.org/10.1590/S010055022011000400016.

3. Marcondes CA, Pessoa SGP, Pessoa BBGP, Dias IS, Guimarães MGM, De Castro SN. Sistematização do treinamento teórico e prático de técnicas em suturas para acadêmicos de medicina da disciplina de cirurgia plástica da Universidade Federal do Ceará - UFC. Rev Bras Cir Plast. 2014;29(2):289-93. http://www.dx.doi.org/10.5935/21771235.2014RBCP0053.

4. Gomes R, Brino RF, Aquilante AG, De Avó LRS. Aprendizagem baseada em problemas na formação médica e o currículo tradicional de medicina: uma revisão bibliográfica. Rev Bras Educ Med. 2009;33(3):444-51. http://dx.doi. org/10.1590/S0100-55022009000300014.

5. Anastakis DJ, Regehr G, Reznick RK, Cusimano M, Murnaghan J, Brown M, Hutchison C. Assessment of technical skills transfer from the bench training model to the 
human model.. Am J Surg. 1999;177(2):167-70. https://doi. org/10.1016/S0002-9610(98)00327-4.

6. Chappell S, Vilke GM, Chan TC, Harrigan RA, Ufberg JW. Peripheral venous cutdown. J Emerg Med. 2006;31(4):411-6. http://dx.doi.org/10.1016/j.jemermed.2006.05.026.

7. Marques RG. Técnica operatória e cirurgia experimental. Rio de Janeiro: Guanabara Koogan; 2005. p.241-5.

8. Carlotti APCP. Acesso vascular. Medicina (Ribeirão Preto). 2012;45(2):208-14. http://dx.doi.org/10.11606/issn.21767262.v45i2p208-214.

9. Comitê do Trauma do Colégio Americano de Cirurgiões. Suporte avançado de vida no trauma para médicos: manual do curso de alunos. 8a ed. Chicago: American College of Surgeons; 2008. p.71-2.

10. Guimaraes Filho, Vaz FA. Apresentação de um novo modelo sintético para treinamento em neuroendoscopia [tese]. São Paulo: UNIFESP; 2012. Disponível em: http://repositorio. unifesp.br/handle/11600/22812.

11. Dantas AK. Avaliação do aprendizado em técnica cirúrgica empregando três estratégias de ensino [tese]. São Paulo:

Artigo recebido em: 10.04.17

Artigo aceito em: 02.08.17
Faculdade de Odontologia, Universidade de São Paulo; 2010. doi: 10.11606/T.23.2010.tde-28052010-125748.

12. Netto FACS, Sommer CG, Constantino MM, Cardoso M, Cipriani RFF, Pereira RA. Projeto de ensino: modelo suíno de baixo custo para treinamento de drenagem torácica. Rev Col Bras Cir. 2016;43(1):60-3. http://dx.doi.org/10.1590/010069912016001012.

13. Ault MJ, Rosen BT, Ault B. The use of tissue models for vascular access training. Phase I of the procedural patient safety initiative. J Gen Intern Med. 2006;21(5):514-7. doi: 10.1111/j.1525-1497.2006.00440.x.

14. Garrood T, Iyer A, Gray K, Prentice H, Bamford R, Jenkin R, Shah N, Gray R, Mearns B, Ratoff JC. A structured course teaching junior doctors invasive medical procedures results in sustained improvements in self-reported confidence. Clin Med. 2010;10(5):464-7. doi: 10.7861/clinmedicine.10-5-464.

15. Miranda RB, Nardino EP, Gomes T, Farias P. Nova técnica para treinamento em acessos vasculares guiados por ultrassom utilizando modelo de tecido animal. J Vasc Bras. 2012;11(1):83-7. http://dx.doi.org/10.1590/S167754492012000100015 . 